\title{
INTENTIONALITY, INFORMATION, AND EXPERIENCE
}

JOHANNES L. BRANDL

University of Salzburg

\section{INTRODUCTION}

Descartes claimed that the essence of the mind is thinking (cogitare), to which Brentano added that the essence of thinking is to be mentally directed at objects of some kind. ${ }^{1}$ These are controversial assumptions about the nature of the mind. A more modest starting point would be to say that intentionality is an important feature of a large class, but not necesarrily of all mental phenomena. Though this claim too has been challenged in the behaviourist tradition, it is now widely accepted that having a mental life involves mental states with a mental content. Cognitive scientists call it the representational power of the mind. Like Descartes and Brentano, we therefore face the task of explaining this important feature of the mind. Where does its representational power come from?

It is also widely agreed today that the project of reducing intentionality to language has failed. The representational power of the mind does not derive from our capacity to speak a language. It is rather the other way round: linguistic expressions derive their meaning and their referential power from the mental states of speakers and hearers which guide their linguistic behaviour. Mental representation is the foundation of linguistic representation. The foundations on which the power of mental representation rests must lie elsewhere. Can we dig here any deeper? Sceptical philosophers like Quine have resisted that demand. That, however, is not a comfortable position, if one has agreed that intentionality is a real feature of thought. How could there be no further explanation of how the mind acquires its representational power?

\footnotetext{
${ }^{1}$ Brentano gives credit here to the scholastic doctrine of "intentional (or mental) inexistence", i.e. the existence of objects "in" psychic phenomena (see Brentano 1874/ 1973, p.88). Though the term "intentionality" derives from Brentano and therefore still echoes these historical roots, the term "object" no longer denotes what exists "in" mental acts but has been replaced by the term "mental content".
} 
Fortunately, there is a better option available. In fact there are two broadly conceived programs for explaining intentionality that compete with each other. One of them is informational semantics. ${ }^{2}$ It takes the category of information to be fundamental and tries to explain how intentional mental states arise in cognitive systems from tracking the information that is available in their environment. Theories that propose to naturalize intentionality typically follow this program. The other project might be called phenomenological semantics. ${ }^{3}$ It rests on the claim that intentionality is founded in conscious experience. Accordingly it tries to explain how conscious experience generates a phenomenal content from which the conceptual content of thoughts can be derived. This too might be seen as providing intentionality with a natural foundation, but it is clearly a very different form of "naturalization". Thus we face a difficult choice: where should we put our money?

The goal of this paper is modest in several ways. First, I will not argue for the claim that informational semantics and phenomenological semantics are the only two games in town. I do think, however, that on a broad understanding of these terms most theories of intentionality may be regarded as belonging to one of these frameworks.

Secondly, I cannot rule out the possibility that there is a deeper level of explanation at which informational semantics and phenomenological semantics might be reconciled with each other. There are theories, like Castañedas guise theory, that might be interpreted along these lines. ${ }^{4}$ However, I think that such an integrative theory, if it is successful, would be more like a third approach that shares some features with the others but also gives up some of their basic assumptions.

\footnotetext{
2 I use the label 'informational semantics' here in a broad sense to include any theory that assigns a fundamental role to natural meaning. This holds for informational theories that emphasize causal relations, like the semantics 'Wisconsin style' of D. Stampe, B. Enç and F. Dretske, as well as for other naturalistic accounts of mental representation by A. Denkel, R. Millikan, D. Papineau, K. Sterelny, and others (see Macdonald \& Papineau 2006).

${ }^{3}$ I use the label 'phenomenological semantics' here for any theory that assigns a fundamental role to experience in the constitution of mental content. This includes Husserl's theory of meaning constituting acts, but also a conceptual role semantics that starts with phenomenal intentionality, and other recent contributions to the 'phenomenal intentionality research project' by T. Horgan, U. Kriegel, B. Loar, G. Strawson, and others (see Bayne \& Montague, forthcoming).

${ }^{4}$ More recently, Edward Zalta has suggested that his theory of abstract objects might allow for a similar reconciliation; see Zalta 2000.
} 
Thirdly, I am not going to suggest a method or criterion of how one might rationally choose between informational semantics and phenomenological semantics. Clearly, both programs have their advantages and disadvantages, and it would take considerably more space than is available here to evaluate them and weigh their respective pros and cons.

The modest task I set myself here is to show how the conflict between informational semantics and phenomenological semantics cannot be resolved. It cannot be resolved, so I shall argue, by demonstrating that one of these programs is "deeply flawed" or perhaps even inconsistent. Arguments that try to find such a flaw in informational semantics have been proposed by Jonathan Lowe (1995/97) and Uriah Kriegel (2007). These arguments, so I shall argue, are ultimately question-begging.

In sections 1 and 2 I do the stage-setting by introducing the main ideas and the attractive features that one finds in informational semantics and phenomenological semantics respectively. In section 3 I present and criticize Lowe's argument against informational semantics. A brief summary of the core of Kriegel's argument is given in section 4, followed by my criticism of this argument in section 5. In the final section I indicate how these results may be helpful - even if not decisive - in finding the right method for explaining intentionality.

\section{FROM INFORMATION TO INTENTIONALITY}

Information is a commodity of our daily life. We continuously receive, transmit and store information of all kinds. Although this information processing is very familiar to us, it is hard to say exactly what this comes to. What is this curious thing called 'information' that exists in our brains, in books and on TV, in our computers, and in many other places?

An answer to this question has been offered by Fred Dretske in his book Knowledge and the Flow of Information (1981). This seminal work launched the project of informational semantics in explaining the foundations of cognition. Dretske's conception of information takes its lead from Claude Shannon's probabilistic notion of 'amount of information' that a signal can carry in a communication process. It diverges from Shannon, however, by also appealing to the semantic notions of 'meaning' and 'content'. Thus Dretske arrives at a definition of informational content. It defines the content that a signal $s$ carries, relative to the knowledge $k$ of an agent who receives that piece of information. The definition says: "A sig- 
nal $r$ carries the information that $s$ is $F={ }_{\mathrm{df}}$ The conditional probability of $s$ 's being $F$, given $r$ (and $k$ ), is 1 (but, given $k$ alone, less than 1.)" (Dretske 1981, p.65).

This technical notion seems at first sight to be far removed from our commonsensical understanding of information. We take it that a piece of information can be more or less accurate and that a person can have misleading or even completely wrong information. This is not compatible with Dretske's definition. It is therefore a bit surprising when he claims that his notion of informational content "corresponds strikingly well with our ordinary, intuitive understanding of information: [...] it enables us to understand the source [...] of the semantic character of information; and it reveals the extent to which, and the reason why, the information one receives is a function of what one already knows." (Dretske 1981, pp.81f.) Where do we find the correspondence that Dretske is speaking of here?

The intuitions that fit Dretske's proposal can be found in the veridical usage of terms like 'perception', 'remember' and 'know'. This usage is constrained by the condition that a subject $S$ can perceive an object $O$, or perceive that $O$ has a certain property $F$, only if $O$ exists and actually has that property. Equally, one can remember that something happened only if it really happened, and one can know something only if it is true. In these contexts we use the notion of informational content (or simply 'information') in the way in which Dretske defines it. We say, for instance, that in perceiving something we pick up information, that in remembering something, we retrieve information from our memory, and that a person who is thus informed about a subject matter knows something about it.

The point here is that perception and memory bring us in contact with reality. This contact becomes more elusive when we consider mental operations in general, not just cognitive operations like veridical perceptions and successful cases of memory retrieval. The faculties of perception and memory can also deceive us. They may deceive us about being in contact with reality. In this case we still have thoughts and these thoughts are about reality, but in a different sense: they merely purport to bring us in cognitive relation with real objects and states of affairs. They create an appearance of contact that is not actually there.

Informational semantics requires acknowledging the fact that mental states can deceive us, while also respecting the veridical usage of epistemic terms. In this way the Dretskian notion of information can be sustained. The insight here is that carrying information (in the Dretskian sense) is not essential for a mental state, just as it is not essential for it to bring us in 
contact with reality. This insight gives rise to an important question: Why is it that cognitive systems are necessarily fallible? Why is it that any system that has the power to receive and store information by perceiving and remembering things, also has the power of forming false beliefs and false memories about reality?

This question takes us beyond the notion of information, as Dretske defines it. The central term now becomes 'representation'. With this notion we enter the familiar territory of a theory of intentionality. The fallibility of mental operations is their central feature from an intentional point of view. There are three aspects to it that may be distinguished:

(1) Thoughts can represent existing objects as well as objects that do not exist.

(2) Thoughts can represent some object $O$ that has property $F$ without representing the fact that $O$ has $F$.

(3) Thoughts can represent some object $O$ although the subject of this thought does not believe to have thoughts about $O$.

How does informational semantics explain these features? That question is too complex to be answered succinctly. There are several attractive ideas that one may pursue here. I can only mention some of these ideas without offering any details.

First, there is the idea of teleological function and cognitive fitness championed in the work of Ruth Millikan (see Millikan 1989/93). According to this idea, thoughts have the power to represent because in doing so they enhance the cognitive fitness of the system in which they occur. For instance, it is of great importance for an organism to know when an enemy is near. It is therefore part of the proper function of its cognitive apparatus to make the system aware of the presence of enemies. Yet objects may appear to be dangerous for the system without actually being so. When the system is thus deceived, its cognitive system still performs its proper function of indicating the presence of a dangerous object. This is, in a nutshell, the teleological explanation how our thoughts can come to represent nonexisting objects.

A second idea is "nomological control". Jerry Fodor has proposed this idea for explaining which properties enter the content of a concept and thus become part of the thoughts we have about objects having those properties (see Fodor 1990). For instance, why do we think of an animal with a tail when we think of a dog? This cannot be explained by saying that all 
dogs that we have seen actually had tails since we may have seen dogs without tails. Yet we might try the following explanation: a concept $C$ refers to objects with a certain feature $F$ iff $C$ is under the nomological control of $F$, i.e. if there is a counterfactual-supporting causal relation between tokens of $C$ and instances of $F$. This might explain why "having a tail" is part of our concept of dog and why other features, like their color, are not. This is, very briefly stated, the nomological explanation of how we can represent objects without also representing all the properties they have.

A third idea that plays an important role in informational semantics is etiology. This idea may be used to explain why our thoughts can represent objects different from those that we believe them to represent. Such cases have become prominent with the Twin-Earth thought-experiments. My twin on Twin-Earth may believe that he is drinking water and that he has thoughts about water, while in fact he is drinking and thinking about a quite different substance. Causal theories of reference explain this possibility along the following lines: A concept $C$ refers to objects of kind $K$ iff an object of kind $K$ has been the incipient cause for forming the concept $C$. That explanation is useful also in cases of real life. We all have many false beliefs about the environment we live in. This need not prevent us from having thoughts about the real substances we interact with. Only etiology, so it seems, can explain this peculiar aspect of intentionality.

These ideas have often been taken to be in competition with, or even opposed to informational semantics (see Fodor 1990; Millikan 1990/93). If one thinks of it as a project that addresses a number of different problems, however, these ideas may also be fruitfully combined with each other. Explanatory power can thus be gained by combining a teleological background theory with the facts of nomological co-variation and incipient causation. This certainly looks like a highly promising research program for a reductive explanation of intentionality. ${ }^{5}$

But doubts remain. These doubts do not just concern the details of the program. There are reasons to think that informational semantics might be completely on the wrong track. Before I consider some of these objections, however, I want to introduce the main alternative approach to explaining intentionality, namely phenomenological semantics.

\footnotetext{
${ }^{5}$ For a recent example of such a combined approach see Jesse Prinz 2002.
} 
Phenomenological semantics takes its inspiration from the work of Brentano. As I mentioned at the beginning, Brentano claimed that to be directed at an object is an essential feature of all mental phenomena. He therefore conjectured that even the most primitive experiences that we find in a human mind already exhibit a form of intentionality. This prompted him to take the notion of "being directed at an object" in a very broad sense. These objects may be common things like an apple that we see or a doorbell that we hear ringing. We should also include in the class of objects, however, such entities as colors and sounds. One might call them "objects of appearance" that we encounter in experience. ${ }^{6}$

In this way Brentano made the simplest experiences - he calls them 'simple presentations' - the starting point for a theory of intentionality. There was a special reason for him to take this approach. Brentano was interested in psychology as a discipline that could play a fundamental role for scientific inquiry in general, including philosophy. This could not be a psychology that relied on observations of behaviour and brain functions. Hence, Brentano began to develop what he later called a descriptive psychology that operates from a first-person perspective. Its task is to give a comprehensive description of the presentations that form the basis of our consciousness and also to provide an account of how complex mental phenomena are founded on simple experiences. If this is done strictly from the first-person perspective, Brentano thought, it will lead to descriptions of mental phenomena that are immediately evident. These descriptive truths can then be taken as a starting point and as background knowledge that is needed in philosophy as well as in the experimental sciences.

What exactly is the role that intentionality plays in this project? There are two ways in which we might interpret Brentano's project. One reading would be that intentionality is for Brentano an irreducible feature of experience that cannot - and need not - be explained any further. It is an immediately evident truth of descriptive psychology that experiences are object-directed by their very nature. Intentionality would thus be given to us as a fundamental feature of experience. It would make no sense here to ask where this power of representing objects comes from. Intentionality is

\footnotetext{
${ }^{6}$ Brentano calls these objects "physical" phenomena thereby indicating that they belong to the physical world at which our mind is directed (see Brentano 1874/1973, pp.79f.).
} 
simply "there" as part of the experience and thus has to be presupposed in every explanation of mental phenomena.

Another possible interpretation, however, opens up when we add a distinction that one might make between the content of experience and the content of a thought that results from "processing" the experience in a certain conceptual framework. Such a distinction has been commonly made in the Kantian tradition, and it may have been Husserl who first seized the opportunity of connecting this distinction with Brentano's ideas. The payoff is that intentionality now can - and even needs to be - reductively explained. Conscious experience is not itself intentional by nature, but only provides the foundation for intentional content. It is from experience that our thoughts derive the power for mental representation.

This move beyond Brentano also creates a new problem however. How can we describe what an experience is like without describing it as object-directed? Consider, for example, the visual experience of a blinking red light. There is no way how I could articulate what that experience is without saying that it is an experience of a red light that is blinking. I must describe the experience by describing the object at which the experience is directed. How else could I make clear what I am talking about?

Husserl found a congenial solution to this problem. In his Logical Investigations (1901) he draws a firm distinction between the content of an experience and the objects that such an experience may or may not represent. This allows us, Husserl claims, to say that a perceptual experience has a certain phenomenal content without mentioning the fact that it directs the attention to an (external) object. We can bracket the external world in describing the nature of our experiences from a first-person perspective.

Since Brentano also used the term 'content', however, this term now becomes highly ambiguous and much caution is in order here. Following a contemporary usage, one might distinguish between the narrow (phenomenal) content of an experience that is not yet intentional, and the wide (intentional) content of mental states that have a representational function. Unfortunately the term 'wide content' is also used for the target objects that are represented in such states. Alternatively, one might adopt a proposal that distinguishes between the 'intentional content' of experience and the 'cognitive content' of thought (see Prinz 2002, pp.3ff). Given this multitude of usages, I think we need another term here that we can contrast with the term 'content'. The term 'character' comes in handy at this point. One might say that experiences have a phenomenal character that is somehow too basic to be described in representational terms. It is a feature of 
experience that we can access from the first-person point of view, and it is different from intentional content (of all sorts).

How could this appeal to phenomenal character help in a reductive explanation of intentionality? A plausible suggestion here is to draw on insights from developmental psychology. We might learn how phenomenal character is turned into intentional content by studying how children learn to identify and recognize objects in their perceptual field. Jonathan Lowe has endorsed this approach:

The intentional content of a perceptual experience is, in a certain sense, grounded in its phenomenal character, but that grounding relation here is a complicated one, which arises at least in part through the subject's individual history of perceptual learning. (Lowe, 1995/97, p.118).

Lowe takes the intentional content of an experience to be the result of our conceiving of objects in a certain way. This is something we learn in infancy, and we do so on the basis of how objects appear to us. Therefore, Lowe concludes, it is a plausible assumption that "how we conceive of physical objects is inextricably bound up with how they appear to us in perception." (ibid.)

This "inextricable" connection between phenomenal character (how things appear to us) and intentional content (how we conceive of them) poses a problem for informational semantics, as Lowe argues. I will deal with this objection shortly. First, however, I want to make explicit what the alternative is. How does a phenomenological explanation of intentionality work?

Phenomenological semantics, too, is a large-scale project. In carrying this project out, three steps need to be taken:

(1) First one has to give a description of appearances as such, i.e. a pure description of their phenomenal character.

(2) Secondly, one has to explain how the intentional content of these experiences is grounded in their phenomenal character.

(3) And thirdly, one has to explain how the conceptual content of thoughts is grounded in the intentional content of perceptual experiences.

The enormous range of this task can be seen from the attempts that have been made to execute such a program in Husserl's constitution systems, or in Carnap's Aufbau-project. One also must not overlook the complication 
that Lowe mentions in the quotation above. The explanation steps here can only be very local since they may be different from concept to concept, and even from subject to subject. For instance, my concept of dog may be grounded in real encounters with dogs, while my concept of a sea gull may be grounded in experiences with picture books. For other subjects it may be just the other way round. This could be a serious difficulty in systematizing the approach and in defining a hierarchy of concepts depending on how closely they are related to experience. But if informational semantics fails, this may be the only way to go.

\section{A “FUNDAMENTAL FLAW" IN INFORMATIONAL SEMANTICS?}

I have now described two different large-scale projects of how the intentionality of mental states may be explained in a reductive manner. According to the first project, intentionality is founded on the faculty of tracking information by cognitive systems that causally interact with their environment. According to the second project, intentionality is founded on the phenomenal character of experience that is accessible to subjects even when they disregard the objects in their environment. The task before us now is to find a way how one can rationally decide between these projects.

Advocates of both projects may point out the specific advantages of their approach. The information-theoretic approach is usually advocated by saying that it naturalizes intentionality (see Loewer 1987). It takes into account that intentionality comes in degrees and exists at different levels. We therefore need to compare simple minds that exhibit only a low grade of intentionality with more complex systems - such as the minds of human beings - that reach a much higher level of intentionality. It also takes into account that intentionality can be found in unconscious mental states, as they occur at a sub-personal level, and that it might be found even in artefacts, like robots, that compute information and thereby adapt to their environment.

This has raised doubts that mental features are here confused with non-mental features. Critics of the information-theoretic approach have thereby turned an alleged advantage of the program into a serious objection against it. Intentionality cannot be found at the biological or computational level, they say. Therefore informational semantics commits a fatal mistake when it tries to explain the intentional content of mental states in terms of the functioning of devices for information processing as they can be found 
in biology or computer science. Much hinges here on the question where to draw the line between an organism that performs certain biological functions and a cognitive system that is capable of having genuine thoughts with intentional content. But why should this be such a decisive question? Why should an advocate of informational semantics not simply reject this question and say that no sharp line can be drawn here?

Jonathan Lowe has therefore tried to strengthen the argument that informational semantics commits a fatal mistake in taking intentionality to arise in simple minds. The mistake here is, Lowe argues, that the project of informational semantics ignores the question whether simple minds have any phenomenal experiences in the first place. If such experiences are missing, no amount of information processing can provide such systems with genuine intentionality.

The point that Lowe is trying to make here is clearly a fundamental one. It is a mistake, Lowe argues, that philosophers have started to separate the problem of explaining intentionality from the problem of explaining phenomenal consciousness. These problems cannot be detached from each other:

The upshot is that it is quite erroneous to suppose that we can ascribe genuine thoughts, with conceptually articulated structure, to creatures or machines lacking altogether the capacity to enjoy conscious experiences with phenomenal or qualitative character. Whatever a computer can do by way of information processing, storage and retrieval is not by any means to be confused with what a thinking human being does who reasons, remembers and recalls. (Lowe 1995/97, p.119)

If Lowe is right, it would be a decisive advantage of the phenomenological project that it takes the subjectivity of mental states to be fundamental. It would be decisive since we are living in a world of appearances that determines all our thinking and reasoning. All thinking takes place from a certain perspective that is defined by how things appear to a single subject. Hence, no explanation of the intentional content of thought can succeed from an objective point of view that transcends our subjective experience.

But like before, this alleged advantage of phenomenological semantics also has a downside. Critics of phenomenology can try to counter this objection by pointing out that their program shows how intentionality can arise from informational processes that do not presuppose a subjective point of view. The phenomenological objection thus takes subjectivity too seriously. It is true that within a certain realm subjectivity reigns, namely 
in the realm of sensory experience. But there is also the realm of representations that are publicly available and not subjectively grounded. Symbolic systems, like languages, are such public symbol systems that allow us to form thoughts whose content is accessible to everyone. It is therefore simply false to claim that all intentional content has to be tied to the perspective of individual subjects.

This dialectic shows, I think, that it is very unlikely to reach a rational decision along these lines. The arguments that are used here all turn out to be question-begging. They do not rely on standards of evaluation that are generally accepted. They are based on premises that are itself part of the debate. One cannot refute the program of informational semantics by taking for granted the premises on which phenomenological semantics is based. And conversely, one cannot refute phenomenological semantics on premises that are central to informational semantics. Such debates will necessarily end in a stale-mate.

In the next section I will consider a different argument that tries to avoid this dialectical impasse.

\section{A NEGLECTED PROBLEM OF ONTOLOGY?}

In a recent paper Uriah Kriegel proposes to evaluate theories of intentionality with respect to their ability to solve a fundamental problem of ontology which he calls "the perennial problem of intentional inexistence" (Kriegel, 2007). This problem has been widely discussed in the tradition of Brentano, but it has played hardly any role in the "mainstream research into intentionality" as it has been pursued since the advance of informational semantics (ibid, p.312). It seems that the problem is no longer considered to be a serious and important problem for a theory of intentionality. But that is a mistake, Kriegel argues, because it is a truly perennial problem that needs to be solved.

Kriegel thus sets aside the debate about naturalizing intentionality. One may subscribe to this goal, he suggests, without agreeing on the way in which it can be achieved. The way in which informational semantics tries to achieve this goal, Kriegel says, "is to identify the natural (broadly causal) relation that holds between $x$ and $y$ when, and only when, $x$ represents $y$." (ibid.) That is where the problem of intentional inexistence can teach us a lesson. It shows that this attempt is ontologically confused and 
that we need a "reconceptualisation of how we are to go about naturalizing representation" (ibid, p.331, fn.13).

So, let us consider the problem of intentional inexistence. Kriegel describes it as a problem that arises from three propositions each of which have a certain prima facie plausibility:

(P1) One can think of (represent) non-existents.

(P2) One cannot bear a relation to non-existents.

(P3) Thinking of (representing) something involves constitutively bearing a relation to it.

Proposition (P1) is plain common sense since we can think of dwarfs and monsters that we hope to be non-existent. It is less obvious that we cannot stand in relation to such creatures by imagining them or by fearing them. But one might argue for the plausibility of proposition (P2) by pointing out that some relations clearly cease to obtain if one of its relata ceases to exist. For instance, a person is no longer married but becomes a widow or widower when his or her spouse dies. Examples like this can be interpreted in different ways, however, and the relation of being married to somebody may be different from the relation of thinking about him. We still seem to be related to people that no longer exist when we stay emotionally attached to them. Thus it seems that proposition (P3) too expresses something that we intuitively accept as true.

Now the problem of intentional inexistence arises as a problem of logical consistency. There is no obvious way of consistently subscribing to all three propositions. Once we accept two of them, we seem to be committed to denying the third proposition in this triad. Hence, we must face the question as to how we can best save our intuitions here without sacrificing our logical consistency.

Kriegel spends some effort on showing how implausible it would be to reject propositions $(\mathrm{P} 1)$ or $(\mathrm{P} 2)$, and he assumes that no serious attempt at reconciling all three propositions can be made. The only option remaining is therefore to deny proposition (P3). The intuition that supports this proposition seems less strong and might therefore be outweighed by the stronger intuitions in favour of the two other propositions. This is the general strategy we must pursue in solving the problem of intentional inexistence.

But this is not the strategy of the information-theoretic approach, Kriegel tells us. The goal in this approach has been, as mentioned above, to 
identify representational facts with the obtaining of a relation that can be described in causal terms. If one accepts that goal, one is committed to "the claim that representing something involves constitutively bearing a relation to it." (ibid, p.312).

This is how far Kriegel develops his argument against informational semantics. In the remainder of the paper he proposes his own solution to the problem of intentional inexistence. This solution I will not discuss here any further, except to mention two important assumptions that Kriegel adds to the intuitions for which he has argued so far. He accepts as a fundamental premise the claim of phenomenological semantics that all intentional content is grounded in the structure of consciousness. And he defends an adverbialist analysis of the intentional content of conscious representations.

These two assumptions are important for the following reason. They are not only fundamental to Kriegel's proposal how we can legitimately reject the idea that representing something involves constitutively bearing a relation to it, i.e. how we can get rid of proposition (3). The two assumptions just mentioned also are needed to complete his argument against informational semantics. The argument, in its completed form, runs as follows:

K1) Information-theoretic explanations of intentionality assume that such explanations must appeal only to natural (broadly causal) relations.

K2) Thinking of non-existing objects cannot be explained by appealing to natural (broadly causal) relations.

K3) The only way to avoid relations to non-existing or merely possible objects is to opt for an adverbialist analysis of conscious representations.

K4) Accepting an adverbialist analysis of conscious experience means to give up the research project of informational semantics in favour of the program of phenomenological semantics.

Once the argument is set out completely in this form, one sees how much depends here on giving a proper solution to the problem of intentional inexistence. Initially it was a logical puzzle that required some adjustment in our intuitions. Now, it seems that in making these adjustments we are driven to a fundamental decision about the general form that a semantic theory can take. 
But the argument fails, I think, both in its initial stage as well as in its completed form. Information-theoretic semantics is not committed to accepting proposition ( $\mathrm{P} 3$ ), and therefore the adverbialist analysis of conscious representations is not the only way for a theory of intentionality to avoid relations to non-existing objects. This is what I shall argue for in the next section.

\section{INFORMATIONAL SEMANTICS REMAINS UNDEFEATED}

Advocates of informational semantics have not been much concerned about the problem of intentional inexistence. In Kriegel's view this has been a dangerous neglect. Reflecting on this problem can show us that it is a misguided attempt to combine a naturalistic ontology with a relational treatment of intentionality. These two goals are simply incompatible. One will either miss the first goal, because one must tacitly accept in one's ontology non-existent or possible objects; or one will fail the second goal because one must give up the relational view inherent in informational semantics in favour of what he calls "phenomenal adverbialism". 7

This dilemma opens up, however, only if one interprets the relational view of intentionality in a highly unfavourable way. Kriegel describes this view as saying that there is a certain relation $R$ that holds if and only if an item $x$ represents an object $y$. It should therefore be possible to identify the obtaining of a representational fact with the obtaining of a relation that can be described in broadly causal terms. But the claims that are actually made by informational semantics are considerably weaker. The relational analysis applies only to signals that carry and transmit information about some object $O$, not to all processes or states with a representational function. Therefore, there is an additional explanatory job to be done here. One needs to explain how the representational power of a mental state can be grounded in the informational relations that are taken as fundamental. It is to be grounded in such relations, but not to be identified with them.

A proper description of informational semantics therefore has to distinguish two levels on which this theory operates: there is (a) the level of relations that enable cognitive systems to pick up and transmit information,

${ }^{7}$ This is not strictly correct since adverbialism could also be developed in a nonphenomenological manner, for instance in terms of conceptual role semantics. Kriegel mentions this possibility on another occasion, but does not discuss it further (see Kriegel 2008). 
and there is (b) the level of cognitive functions that enable such systems to represent objects, properties, and facts. At the bottom level, information can be transmitted only if certain (broadly causal) relations obtain. At the cognitive level systems can perform cognitive functions even if the objects that they represent do not exist. Those functions may not be "proper" functions in the biological sense of serving a biological purpose. There may be other purposes that make it useful for a system to have representational powers that transcend the realm of what actually exists.

If one conceives of informational semantics in this way, how should one respond to the logical puzzle that arises from the problem of intentional existence? The solution will be just what Kriegel suggests, namely to give up proposition (P3) in his depiction of the puzzle. This proposition (P3) says that thinking of (representing) something involves constitutively bearing a relation to it. Kriegel argues, as we have seen, that informational semantics is committed to the truth of this proposition. But he can claim this only by ignoring the two levels on which informational semantics operates. It would be perfectly correct to say that perceiving an object or remembering a certain event involves constitutively bearing a relation to the perceived object or the remembered fact. It would be correct because perception and memory are veridical in nature. They require that information is transmitted from the objects and events to the subject who perceives or remembers them. It is the veridicality of those states, not their representational function, that needs to be explained in relational terms. Since mental states are often non-veridical, however, proposition (P3) cannot be true as it stands.

We can see now why informational semantics has not been much concerned with the puzzle of intentional inexistence. If one takes the distinction between veridical and non-veridical states to be fundamental, it becomes difficult to see why there should be a problem here in the first place. When the puzzle is formulated, one immediately suspects that there is something "fishy" about proposition (P3). This proposition is much too general and needs to be restricted in the following way:

(P3*) Perceiving and remembering something involves constitutively bearing an information-transmitting relation to what is perceived and remembered. 
While informational semantics is committed to $\left(\mathrm{P} 3^{*}\right)$, it can safely reject the much stronger claim (P3). Thus we may conclude that informational semantics remains undefeated by the problem of intentional inexistence.

This result also undermines the more elaborate argument against informational semantics that I have tried to reconstruct from Kriegel's paper. This argument starts out from claiming (K1) that only natural relations should be used in explaining intentionality, and (K2) that thinking of nonexistent objects cannot be explained by appeal to such relations. Properly understood, these premises should not be in dispute. A proper understanding of these premises, however, would allow for an appeal to the level of cognitive functions in explaining intentionality. It would be an unreasonable constraint to require that informational semantics has to explain intentionality exclusively on the level of informational relations. Admittedly, there is no way how one could explain at that level how one can think of non-existent objects. But there is also no need to restrict oneself to that level. Thinking of objects - existing or non-existing - may be explained by cognitive functions that are grounded in informational relations. The goal of informational semantics is to explain how this "grounding" of cognitive functions in informational relations gives rise to intentional mental states. The general idea, as we have already seen, is this:

The cognitive function of mental states is grounded in the processing of information in the following way: (a) the intentional content of perceptions and memories is grounded in information-transmitting relations, and (b) the intentional content of all other mental states derives from the intentional content of perceptions and memories.

Does Kriegel's argument provide any reason against pursuing this goal? The argument, as I have reconstructed it, continues with the claim (K3) that the only way to avoid relations to non-existing or merely possible objects is to opt for an adverbialist analysis of conscious representations. With this claim, one simply denies that there exists an alternative project here, namely informational semantics. This project does not take conscious representations to be fundamental, nor does it require an adverbialist analysis of such representations. At this point, Kriegel's argument simply assumes that intentionality has to be grounded in conscious experience. If one takes this basic presupposition of phenomenological semantics on board, however, the objection to informational semantics becomes question-begging again. 


\section{CONCLUSIONS}

In this paper I have tried to make explicit a basic conflict between two large-scale projects concerning how intentionality might be explained. Both projects agree that there are basic facts about the mind that may serve as an explanatory basis, but they disagree what these basic facts are. For informational semantics these foundational facts are given with the cognitive functions of the mind and the information-transmitting relations on which these functions are based. For phenomenological semantics the basic facts are given with conscious experience, with its phenomenal character and with an intentional content that arises from these phenomenal features.

I then considered the problem of deciding which of these programs one should adopt. Here my conclusions have been exclusively negative. I have examined arguments that claim to find a fundamental "flaw" in the project of informational semantics. Examination of these arguments, however, reveals that these arguments either misconstrue the project of informational semantics, or simply assume the truth of some controversial phenomenological premise. No rational decision can be based on such question-begging arguments.

How should one proceed then? A pessimistic conclusion at this point would declare the sceptic to be the winner in this conflict. If we are unable to decide the conflict on rational grounds, the most reasonable thing to do might be to reject some of the premises on which both sides agree. Perhaps we should give up the idea that intentionality is an important feature of the mind, or perhaps we should resist the idea that it can be explained by appeal to certain basic facts about the mind.

My own view is less pessimistic and more pragmatic. I think that we have reason to pursue a semantic project as long as it raises interesting questions and objections that can be answered. This test has been applied here to informational semantics, and so far the project has passed this test. But one must not forget that it is large-scale project. If one asks, for instance, how our thoughts about abstract objects or fictional characters are grounded in perceptual processes by which we pick up information from the environment, an advocate of this project will have a hard time proving his case. Advocates of phenomenological semantics might claim that it is much easier and therefore more reasonable to ground such thoughts in the phenomenal qualities of our imaginatory experiences. That however remains to be seen. 


\section{ACKNOWLEDGEMENT}

This paper was written as part of the European Science Foundations EUROCORES Programm CNCC, and was supported by the Austrian Science Fund (FWF), project I94-G15.

\section{REFERENCES}

Bayne, Timothy and Michelle Montague (eds.) forthcoming. Cognitive Phenomenology. Oxford: Oxford University Press.

Brentano, Franz 1874/1973. Psychology From an Empirical Standpoint. Engl. transl.: London: Routledge.

Dretske, Fred 1981. Knowledge and the Flow of Information. Cambridge Mass.: MIT Press.

Fodor, Jerry 1990. A Theory of Content I \& II. In: J.A. Fodor, A Theory of Content and Other Essays. Cambridge: The MIT Press, pp.89-136.

Kriegel, Uriah 2007. "Intentional Inexistence and Phenomenal Intentionality", Philosophical Perspectives 21: pp.307-340.

Kriegel, Uriah 2008. "The Dispensibility of (Merely) Intentional Objects" Philosophical Studies 141: pp.79-95.

Loewer, Barry 1987. "From Information to Intentionality," Synthese, 70: pp.287-317

Lowe, E. Jonathan 1995/97. "There Are No Easy Problems of Consciousness", reprintetd in: J. Shea (ed.): Facing Up to the Hard Problem of Consciousness, Cambridge Mass., pp.117-123.

Macdonald, Graham and David Papineau (eds.) 2006. Teleosemantics. New Philosophical Essays. Oxford: Oxford University Press.

Millikan, Ruth G. 1989/93. "Biosemantics", reprinted in R.G. Millikan: White Queen Psychology and Other Essays. Cambridge Mass.: The MIT Press, pp.83-101.

Millikan, Ruth G. 1990/93. "Compare and Contrast Dretske, Fodor, and Millikan on Teleosemantics", reprinted in R.G. Millikan: White Queen Psychology and Other Essays. Cambridge Mass.: The MIT Press, pp.123-133.

Prinz, Jesse J. 2002. Furnishing the Mind. Concepts and Their Perceptual Basis. Cambridge Mass.: The MIT Press.

Zalta, Edward N. 2000. "The Road Between Pretense Theory and Abstract Object Theory", in: A. Everett and T. Hofweber (eds.), Empty Names, Fiction, and the Puzzles of Non-Existence. Stanford: CSLI Publications, pp.117-147. 
